Document downloaded from:

http://hdl.handle.net/10251/172321

This paper must be cited as:

Bonet Solves, JA.; Mangino, EM. (2020). Associated weights for spaces of p-integrable entire functions. Quaestiones Mathematicae. 43(5/6):747-760.

https://doi.org/10.2989/16073606.2019.1605420

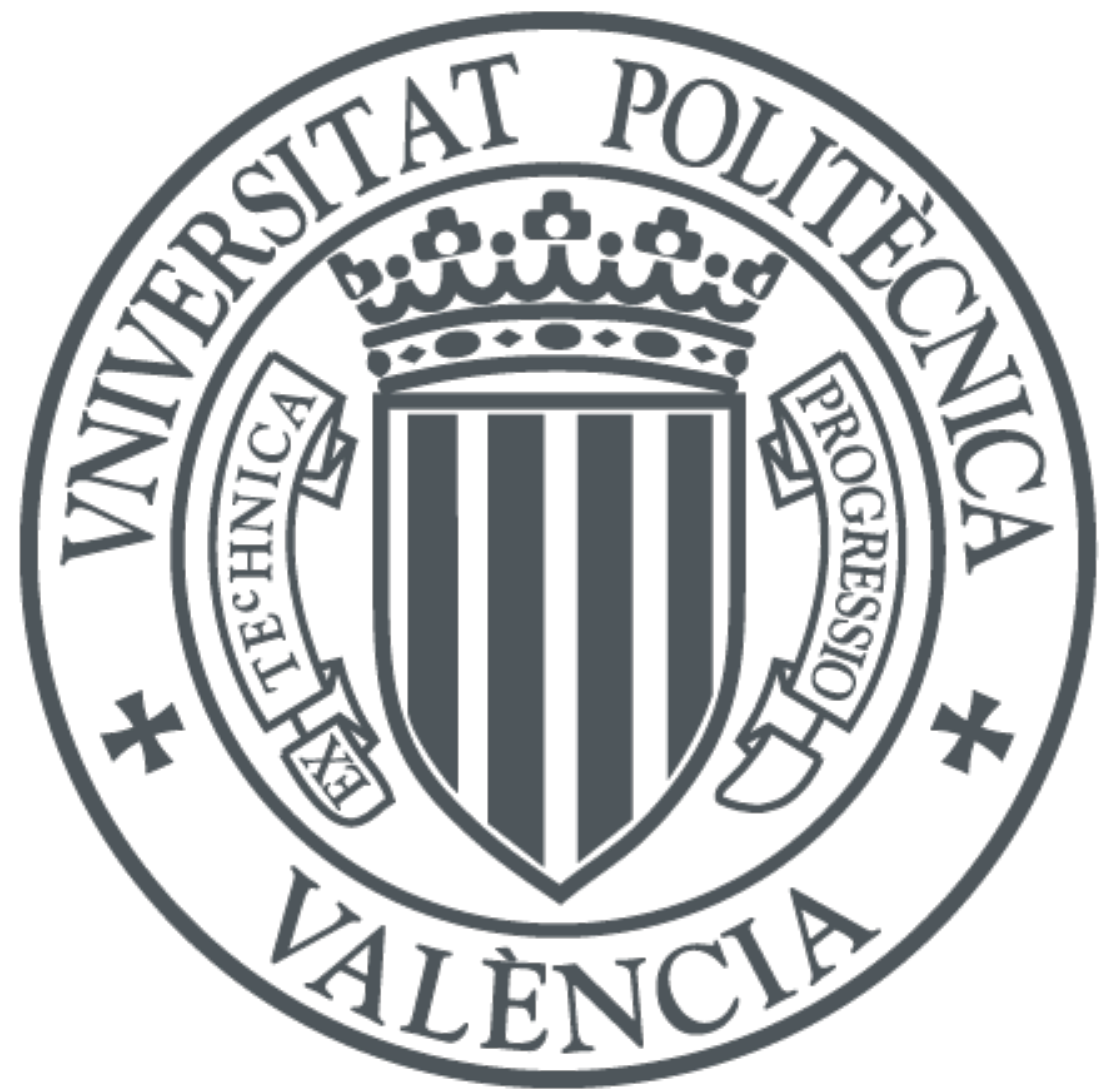

The final publication is available at

https://doi.org/10.2989/16073606.2019.1605420

Copyright Informa UK (National Inquiry Services Center)

Additional Information 


\title{
ASSOCIATED WEIGHTS FOR SPACES OF $p$-INTEGRABLE ENTIRE FUNCTIONS
}

\author{
JOSÉ BONET AND ELISABETTA M. MANGINO
}

\begin{abstract}
In analogy to the notion of associated weights for weighted spaces of analytic functions with sup-norms, $p$-associated weights are introduced for spaces of entire $p$-integrable functions, $1 \leq p<\infty$. As an application, necessary conditions for the boundedness of composition operators acting between general Fock type spaces are proved.
\end{abstract}

\section{INTRODUCTION}

Weighted $L^{p}$-spaces of entire functions, $1 \leq p<\infty$, arise in several different settings, such as partial differential equation, Fourier analysis, and spectral theory. In particular, the Fock $L^{2}$-space (also known as Bargmann space or Segal-Bargmann space) plays an important role in quantum physics (see [2, 21], see also [15, Chapter I, Sec. 6] for an historical introduction).

Properties of composition operators acting between these spaces have been extensively studied by several authors in the last two decades (see $[1,10,17,24,25,26,27$, $28,29]$ ).

There are essentially two approaches to the analysis of weighted $L^{p}$-spaces of entire functions and of composition operators. The first approach has been developed for $p=\infty$ and relies on the study of associated growth conditions, namely weights that are intrisically defined for the space of entire functions under consideration (see, e.g. the seminal paper [4]). The other approach has been initially applied in the case $p=2$ and then extended to $1 \leq p<\infty$, and involves the use of complex and functional analysis techniques, such as representing kernels, Carleson measures, sampling and interpolation sequences.

The aim of this note is to present a connection between these techniques, by introducing an analogous concept of associated weights for $1 \leq p<\infty$. The boundedness of composition operators is then studied by applying $p$-associated weights. Moreover, in the particular case of radial weights, we give conditions on the weights in order that bounded composition operators have to be generated by affine maps, thus extending the classical result that holds true for composition operators between Fock spaces.

\section{NotATION AND PRELIMINARIES}

Consider the space $\mathbb{C}^{N}$ endowed with the complex inner product and the norm defined by:

$$
\langle z, w\rangle=\sum_{i=1}^{N} z_{i} \overline{w_{i}}, \quad|z|=\sqrt{\langle z, z\rangle}, \quad z=\left(z_{1}, \ldots, z_{N}\right), w=\left(w_{1}, \ldots, w_{N}\right) .
$$

Key words and phrases. p-associated weights, Fock type spaces, composition operators. Mathematics Subject Classification 2010: Primary 47B33; Secondary 46 E15. 
We denote by $\mathcal{H}\left(\mathbb{C}^{N}\right)$ the space of entire functions and we consider the usual Lebesgue measure on $\mathbb{C}^{N}=\mathbb{R}^{2 N}$. We will use the notation $\mathcal{H}\left(\mathbb{C}^{N} ; \mathbb{C}^{N}\right)$ for the space $\left(\mathcal{H}\left(\mathbb{C}^{N}\right)\right)^{N}$.

Let $v$ be a weight on $\mathbb{C}^{N}$, namely a continuous function $\left.v: \mathbb{C}^{N} \rightarrow\right] 0, \infty[$. Define for $1 \leq p<\infty$

$$
\mathcal{F}_{v}^{p}=\left\{\left.f \in \mathcal{H}\left(\mathbb{C}^{N}\right)\left|\int_{\mathbb{C}^{N}}\right| f(z)\right|^{p} v^{p}(z) d z<\infty\right\}
$$

and

$$
\|f\|_{p, v}=\left(\int_{\mathbb{C}^{N}}|f(z)|^{p} v^{p}(z) d z<\infty\right)^{\frac{1}{p}}, \quad f \in \mathcal{F}_{v}^{p} .
$$

The spaces $\mathcal{F}_{v}^{p}$ are subspaces of $L^{p}\left(\mathbb{C}^{N}, v^{p} d z\right)$, hence are normed spaces. If $p=2$ the norm is induced by the inner product:

$$
(f, g)_{v}=\int_{\mathbb{C}^{N}} f(z) \bar{g}(z) v^{2}(z) d z \quad f, g \in \mathcal{F}_{v}^{2}
$$

If $p=\infty$ we consider

$$
\mathcal{F}_{v}^{\infty}=\left\{f \in \mathcal{H}\left(\mathbb{C}^{N}\right)\left|\sup _{\mathbb{C}^{N}}\right| f(z) \mid v(z)<\infty\right\},
$$

endowed with the norm

$$
\|f\|_{\infty, v}=\sup _{\mathbb{C}^{N}}|f(z)| v(z), \quad f \in \mathcal{F}_{v}^{\infty} .
$$

The space $\mathcal{F}_{v}^{\infty}$ is often also denoted by $H_{v}^{\infty}\left(\mathbb{C}^{N}\right)$. It is a Banach space and has been widely studied (see e.g. $[3,4,16,18]$ and the references therein).

If $v(z)=e^{-\frac{\alpha}{2}|z|^{2}}$, then $\mathcal{F}_{v}^{p}$ is the classical Fock space $\mathcal{F}^{p, \alpha}$ (see e.g. the monograph [30]).

Lemma 1.1. Let $\left.v: \mathbb{C}^{N} \rightarrow\right] 0, \infty[$ be a continuous function and $1 \leq p<\infty$. For every compact $K \subseteq \mathbb{C}^{N}$, the evaluation map

$$
\delta_{z}: \mathcal{F}_{v}^{p} \rightarrow \mathbb{C}, \quad f \mapsto f(z)
$$

is continuous uniformly with respect to $z \in K$. As a consequence, $\mathcal{F}_{v}^{p}$ is a Banach space and $\mathcal{F}_{v}^{2}$ is a Hilbert space.

Proof. Since every compact subset in $\mathbb{C}^{N}$ is covered by a finite number of polydisks, it is enough to prove that for every $z \in \mathbb{C}^{N}$ there exists $M>0$ such that

$$
\sup _{w \in P(z, 1)}|f(w)| \leq M\|f\|_{p, v}
$$

where $P(z, 1)=\left\{w=\left(w_{1}, \ldots, w_{N}\right) \in \mathbb{C}^{N}|| z_{i}-w_{i} \mid \leq 1, i=1, \ldots, N\right\}$.

Given $z \in \mathbb{C}^{N}$, let $v_{0}$ be the minimum of $v$ on $P(z, 2)$.

Let $f \in \mathcal{F}_{v}^{p}$ and $x \in P(z, 1)$. Clearly $|f|$ is separately subharmonic in each variable, hence for every $r_{1}, \ldots, r_{N}>0$ :

$$
\begin{aligned}
\left|f\left(x_{1}, \cdot, x_{N}\right)\right| & \leq \frac{1}{2 \pi} \int_{0}^{2 \pi}\left|f\left(x_{1}+r_{1} e^{i \theta_{1}}, x_{2}, \ldots, x_{N}\right)\right| d \theta_{1} \leq \cdots \leq \\
& \leq \frac{1}{(2 \pi)^{N}} \int_{[0,2 \pi]^{N}} f\left(x_{1}+r_{1} e^{i \theta_{1}}, \ldots, x_{1}+r_{N} e^{i \theta_{N}}\right) d \theta_{1} \ldots d \theta_{N} .
\end{aligned}
$$


By multiplying both sides by $2^{N} r_{1} \cdots \cdots r_{N}$ and by integrating with respect to $r_{1}, \ldots, r_{N}$ on $[0,1]^{N}$, we get that:

$$
\begin{aligned}
& |f(x)| \leq \frac{1}{\pi^{n}} \int_{P(x, 1)}|f(w)| d w \leq \frac{1}{\pi^{n}} \int_{P(z, 2)}|f(w)| d w \leq \frac{1}{v_{0}} \frac{1}{\pi^{n}} \int_{P(z, 2)}|f(w)| v(w) d w \\
\leq & \frac{1}{v_{0}} \frac{1}{\pi^{n}}\left(\int_{P(z, 2)}(|f(w)| v(w))^{p} d w\right)^{1 / p}\left(\int_{P(z, 2)} d w\right)^{1 / p^{\prime}} \leq \frac{1}{v_{0}} \frac{1}{\pi^{n}}(4 \pi)^{n / p^{\prime}}\|f\|_{p, v} .
\end{aligned}
$$

Finally we prove that $\mathcal{F}_{v}^{p}$ is a closed subspace of $L^{p}\left(\mathbb{C}^{N}, v^{p} d z\right)$. Let $\left(f_{n}\right)_{n}$ be a sequence in $\mathcal{F}_{v}^{p}$ converging to a function $f \in L^{p}\left(\mathbb{C}^{N}, v^{p} d z\right)$. Let $K$ be a compact subset of $\mathbb{C}^{N}$. There exists $C_{K}>0$ such that for every $n, m \in \mathbb{N}$ :

$$
\forall z \in K \quad\left|f_{n}(z)-f_{m}(z)\right| \leq C_{K}|| f_{n}-f_{m} \|_{p, v}
$$

Hence $\left(f_{n}\right)_{n}$ is a Cauchy sequence with respect to the compact-open topology and therefore it converges to an entire function.

Remark 1.2. By the Riesz Representation Theorem, for every $z \in \mathbb{C}^{N}$ there exists $K_{z} \in \mathcal{F}_{v}^{2}$ such that

$$
\forall f \in \mathcal{F}_{v}^{2} \quad \delta_{z}(f)=\left\langle K_{z}, f\right\rangle=\int_{\mathbb{C}^{N}} K_{z}(w) \overline{f(w)} v^{2}(w) d w .
$$

Observe that, for every $z \in \mathbb{C}^{N}$,

$$
\left\|\delta_{z}\right\|_{\left(\mathcal{F}_{v}^{2}\right)^{\prime}}=\sup _{\|f\|_{2, v} \leq 1}|f(z)|=\sup _{\|f\|_{2, v} \leq 1}\left|\left\langle K_{z}, f\right\rangle\right| \leq\left\|K_{z}\right\|_{2, v} .
$$

Considering in particular $f=K_{z} \cdot\left\|K_{z}\right\|_{2, v}^{-1}$, we get that

$$
\left\|\delta_{z}\right\|_{\left(\mathcal{F}_{v}^{2}\right)^{\prime}} \geq\left|\left\langle K_{z}, K_{z}\right\rangle\right| \cdot\left\|K_{z}\right\|_{2, v}^{-1}=\left\|K_{z}\right\|_{2, v} .
$$

Hence

$$
\left\|\delta_{z}\right\|_{\left(\mathcal{F}_{v}^{2}\right)^{\prime}}=\left\|K_{z}\right\|_{2, v}=\sqrt{\left\langle K_{z}, K_{z}\right\rangle}=\sqrt{K_{z}(z)} .
$$

Several examples of spaces $\mathcal{F}_{v}^{p}(\mathbb{C})$ are known in literature. In no way pretending to be exhaustive, we refer to $[1,11,14,19,20,22,23]$. In particular we recall in the next example the spaces $\mathcal{F}_{v}^{p}$ that have been first considered in [23].

Example 1.3. Let $v=e^{-\varphi}$ where $\varphi \in C^{2}\left(\mathbb{C}^{N}\right)$ is a real valued function on $\mathbb{C}^{N}$ such that

$$
m \omega_{0} \leq d d^{c} \varphi \leq M \omega_{0}
$$

holds uniformly pointwise on $\mathbb{C}^{N}$ for some positive constants $m$ and $M$, where $d$ is the usual exterior derivative, $d^{c}=i(\partial-\bar{\partial})$ and $\omega_{0}=d d^{c}|\cdot|^{2}$ is the standard Euclidean Kähler form on $\mathbb{C}^{N}$. The associated spaces $\mathcal{F}_{v}^{p}$ have been thoroughly studied in [23].

If $\varphi(z)=-\frac{\alpha}{2}|z|^{2}$ one gets the classical Fock spaces and for $\varphi(z)=|z|^{2}-m \log |z|$, with $m \in \mathbb{N}$, one gets the Fock-Sobolev spaces $\mathcal{F}^{p, m}$ (see e.g. [12]).

In the case of example 1.3, the following characterization of composition operators holds. For sake of completeness we give a proof with the arguments of [1]. 
Proposition 1.4. Let $v=e^{-\varphi_{1}}$ and $w=e^{-\varphi_{2}}$ with $\varphi_{i} \in C^{2}\left(\mathbb{C}^{N}\right), i=1,2$, real valued functions of the type considered in Example 1.3. Let $\psi \in \mathcal{H}\left(\mathbb{C}^{N} ; \mathbb{C}^{N}\right)$. The composition operator $C_{\psi}: \mathcal{F}_{v}^{p} \rightarrow \mathcal{F}_{w}^{q}, f \mapsto f \circ \psi$ with $1 \leq p \leq q<\infty$, is continuous if and only if there exists $r>0$ (or equivalently for all $r>0$ ) such that

$$
\sup _{z \in \mathbb{C}^{N}} \int_{\psi^{-1}(B(z, r))} \frac{w^{q}(w)}{v^{q}(\psi(w))} d w<\infty .
$$

In particular, if $\frac{w}{v \circ \psi}$ is $q$ - integrable on $\mathbb{C}^{N}$, then $C_{\psi}$ is continuous.

PROOF. Let $\lambda$ be the pull-back measure defined by

$$
\lambda(E)=\int_{\psi^{-1}(E)} e^{-q \varphi_{2}}(z) d z
$$

for any Borel subset of $\mathbb{C}^{N}$. By [1, Section 2, Corollary 3.1], $C_{\psi}$ is a bounded composition operator if and only if the measure $\mu=e^{q \varphi_{1}} \cdot \lambda$ is a $(p, q)$-Carleson measure, or, equivalently by [1, Lemma 2.1$]$, if

$$
\sup _{z \in \mathbb{C}^{N}} \mu(B(z, r))<\infty \quad \text { for some/all } r>0 .
$$

The assertion follows by observing that

$$
\mu(B(z, r))=\int_{B(z, r)} e^{q \varphi_{1}(w)} d \lambda(w)=\int_{\psi^{-1}(B(z, r))} e^{q \varphi_{1}(\psi(z))} e^{-q \varphi_{2}(z)} d z<\infty .
$$

\section{2. $p$-ASSOCIATED WEIGHTS AND COMPOSITION OPERATORS}

Associated weights were introduced in [4], in order to study properties of the space $H_{v}^{\infty}(\mathbb{C})$. Given a continuous weight $v$, the associated weight is defined as

$$
\widetilde{v}(z)=\sup \left\{|f(z)| \mid f \in H_{v}^{\infty}\left(\mathbb{C}^{N}\right),\left(\|f\|_{v, \infty} \leq 1\right\}\right)^{-1} .
$$

It is known that $\widetilde{v}$ is continuous and $0<v \leq \widetilde{v}$ (see [4, Properties 1.2]). The weight $v$ is said to be essential if $v \simeq \widetilde{v}$, namely there exist $c, C>0$ such that

$$
c v \leq \widetilde{v} \leq C v .
$$

We will consider an analogous definition for every $1 \leq p<\infty$.

Definition 2.1. Let $\left.v: \mathbb{C}^{N} \rightarrow\right] 0, \infty[$ be a continuous weight and $1 \leq p<\infty$. The $p$-associated weight $\widetilde{v}_{p}$ is defined by

$$
\widetilde{v}_{p}(z)=\frac{1}{\left\|\delta_{z}\right\|_{\left(\mathcal{F}_{v}^{p}\right)^{\prime}}}, \quad z \in \mathbb{C}^{N}
$$

The weight $v$ is said to be $p$-essential if $v \simeq \widetilde{v}_{p}$.

Remark 2.2. Observe that if $v$ is a 2-essential weight, then, by (1.6),

$$
v(z) \simeq \frac{1}{\sqrt{K_{z}(z)}} .
$$

Example 2.3. Let $v=e^{-\varphi}$ be the weight considered in Example 1.3.

By [23, Corollary 3.7],

$$
f(z)=\delta_{z}(f)=\int_{\mathbb{C}^{N}} K(z, w) \overline{f(w)} e^{-2 \varphi(w)} d w \quad \forall f \in \mathcal{F}_{v}^{p}, z \in \mathbb{C}^{N},
$$


where $K_{z}$ is the reproducing kernel. Then $K_{z} \in \mathcal{F}_{v}^{q}$ for every $1 \leq q \leq \infty$ (see [1, Equ. (2)] $)$ and $\left\|K_{z}\right\|_{q, v} \simeq v(z)^{-1}$. Finally [23, Proposition 3.8] yields that $\left\|\delta_{z}\right\|_{\left(\mathcal{F}_{v}^{p}\right)^{\prime}}=$ $\left\|K_{z}\right\|_{p^{\prime}, v} \simeq v(z)^{-1}$, where $p^{\prime}$ is the conjugate exponent of $p$, and therefore $v$ is $p$ essential.

Example 2.4. Let $v(z)=e^{-|z|^{\alpha}}$, with $\alpha>2, x \in \mathbb{C}$. It has been proved in [14, Corollary 8] that, for large values of $|z|$,

$$
\left\|\delta_{z}\right\|_{\left(\mathcal{F}_{v}^{2}\right)^{\prime}}=\left\|K_{z}\right\|_{2, v} \simeq e^{-|z|^{\alpha}}|z|^{\alpha-2} .
$$

Hence $v$ is not a 2-essential weight.

Remark 2.5. If $v$ is a continuous weight such that $\mathcal{F}_{v}^{p} \hookrightarrow H_{v}^{\infty}$ continuously, then clearly there exists $C>0$ such that $\widetilde{v} \leq C \widetilde{v}_{p}$. Hence, if $v$ is $p$-essential, it is essential. The converse does not hold. For example, the weight $v$ defined as $v(z)=e^{-|z|^{\alpha}}$, with $\alpha>2$, is essential (see e.g. [9, Lemma 1] or [8, Remark 3.11]), but it is not 2-essential.

Proposition 2.6. Let $v$ and $w$ be continuous weights on $\mathbb{C}^{N}, 1 \leq p, q<\infty$, and $\psi \in \mathcal{H}\left(\mathbb{C}^{N} ; \mathbb{C}^{N}\right)$. If the composition operator $C_{\psi}: \mathcal{F}_{v}^{p} \rightarrow \mathcal{F}_{w}^{q}$ is continuous, then

$$
\sup _{z \in \mathbb{C}^{N}} \frac{\widetilde{w}_{q}(z)}{\widetilde{v}_{p}(\psi(z))}<\infty .
$$

Proof. If $C_{\psi}$ is continuous, then

$$
\left(C_{\psi}\right)^{\prime}:\left(\mathcal{F}_{w}^{q}\right)^{\prime} \rightarrow\left(\mathcal{F}_{v}^{p}\right)^{\prime}
$$

is continuous. Hence there exists $C>0$ such that

In particular,

$$
\forall \xi \in\left(\mathcal{F}_{w}^{p}\right)^{\prime} \quad\left\|\left(C_{\psi}\right)^{\prime} \xi\right\|_{\left(\mathcal{F}_{v}^{p}\right)^{\prime}} \leq C\|\xi\|_{\left(\mathcal{F}_{w}^{q}\right)^{\prime}}
$$

$$
\forall z \in \mathbb{C}^{N} \quad\left\|\left(C_{\psi}\right)^{\prime} \delta_{z}\right\|_{\left(\mathcal{F}_{v}^{p}\right)^{\prime}} \leq C\left\|\delta_{z}\right\|_{\left(\mathcal{F}_{w}^{q}\right)^{\prime}}
$$

By observing that

$$
\left(C_{\psi}\right)^{\prime}\left(\delta_{z}\right)(f)=\delta_{z}\left(C_{\psi} f\right)=\delta_{\psi(z)}(f),
$$

we get

$$
\forall z \in \mathbb{C}^{N} \quad\left\|\delta_{\psi(z)}\right\|_{\left(\mathcal{F}_{v}^{p}\right)^{\prime}} \leq C\left\|\delta_{z}\right\|_{\left(\mathcal{F}_{w}^{q}\right)^{\prime}}
$$

therefore there exists a costant $\widetilde{C}>0$ such that

$$
\forall z \in \mathbb{C}^{N} \quad \frac{1}{\widetilde{v}_{p}(\psi(z))} \leq \widetilde{C} \frac{1}{\widetilde{w}_{q}(z)}
$$

As a consequence of the definition of $p$-essential weights and of Remark 2.5, we get.

Corollary 2.7. Let $v$ and $w$ be continuous weights on $\mathbb{C}^{N}, 1 \leq p, q<\infty$, and $\psi \in \mathcal{H}\left(\mathbb{C}^{N} ; \mathbb{C}^{N}\right)$ such that the composition operator $C_{\psi}: \mathcal{F}_{v}^{p} \rightarrow \mathcal{F}_{w}^{q}$ is continuous.

(1) If $v$ is p-essential and $w$ is q-essential, then $\sup _{z \in \mathbb{C}^{N}} \frac{w(z)}{v(\psi(z))}<\infty$.

(2) If $\mathcal{F}_{w}^{p} \hookrightarrow H_{w}^{\infty}$ continuously, then $\sup _{z \in \mathbb{C}^{N}} \frac{w(z)}{\widetilde{v}_{p}(\psi(z))}<\infty$.

Remark 2.8. Proposition 2.6 and Corollary 2.7 should be compared with the characterization of bounded operators between spaces of type $H_{v}^{\infty}$ in terms of associated weights (see [7, Proposition 5]). 


\section{RAdial WEIGHTS}

Motivated by the results in the previous section, we investigate the boundedness of the quotient $\frac{w(z)}{v(\psi(z))}$, where $w$ and $v$ are continuous radial weights on $\mathbb{C}^{N}$ and $\psi \in \mathcal{H}\left(\mathbb{C}^{N} ; \mathbb{C}^{N}\right)$. In the following, if $\left.v: \mathbb{C}^{N} \rightarrow\right] 0, \infty[$ is a radial weight, with an abuse of notation, for any $r>0$ we will write $v(r)$ meaning $v(z)$ for any $z \in \mathbb{C}^{N}$ with $|z|=r$, and we say that $v$ is (strictly) decreasing meaning that $v(r)$ is (strictly) decreasing on $[0, \infty[$.

Proposition 3.1. Let $\left.v, w: \mathbb{C}^{N} \rightarrow\right] 0, \infty[$ be radial continuous decreasing weights and let $\psi \in \mathcal{H}\left(\mathbb{C}^{N} ; \mathbb{C}^{N}\right)$. If for some $\alpha>1$

$$
\lim _{r \rightarrow \infty} \frac{w(r)}{v(\alpha r)}=+\infty
$$

and

$$
\sup _{z \in \mathbb{C}^{N}} \frac{w(z)}{v(\psi(z))}<+\infty
$$

then $\psi$ is affine.

Proof. We show that $\sup _{z \in \mathbb{C}^{N},|z| \geq 1} \frac{|\psi(z)|}{|z|}$ is bounded. Otherwise, there would exist $\left(z_{k}\right)_{k \in \mathbb{N}}$ such that $\left|z_{k}\right| \rightarrow \infty$ and

$$
\forall k \in \mathbb{N}\left|\psi\left(z_{k}\right)\right| \geq k\left|z_{k}\right| .
$$

Then

$$
\sup _{k \in \mathbb{N}} \frac{w\left(z_{k}\right)}{v\left(\alpha z_{k}\right)} \leq \sup _{k \in \mathbb{N}} \frac{w\left(\left|z_{k}\right|\right)}{v\left(k z_{k}\right)} \leq \sup _{k \in \mathbb{N}} \frac{\widetilde{w}\left(z_{k}\right)}{v\left(\psi\left(z_{k}\right)\right)}<+\infty,
$$

against the hypothesis. Now the assertion follows with the argument of [10, Theorem 1]. For sake of completeness we give some hints of the proof. Assume that $\psi=$ $\left(\psi_{1}, \ldots, \psi_{n}\right)$, with $\psi_{i}: \mathbb{C}^{N} \rightarrow \mathbb{C}$. For any $\zeta \in \mathbb{C}^{N},\|\zeta\|=1$, consider the entire function on $\mathbb{C}$

$$
\psi_{i}^{\zeta}(\lambda):=\psi_{i}(\lambda \zeta), \quad \lambda \in \mathbb{C}
$$

If $\psi_{i}(z)=\sum_{s=0}^{\infty} \sum_{|\alpha|=s} c_{\alpha} z^{\alpha}$, since

$$
\sup _{|\lambda| \geq 1} \frac{\left|\psi_{i}^{\zeta}(\lambda)\right|}{|\lambda|}<\infty
$$

for every $\zeta \in \mathbb{C}^{N},\|\zeta\|=1$, we get

$$
\sum_{|\alpha|=s} c_{\alpha} z^{\alpha}=0 \quad s \geq 2
$$

and therefore $\psi_{i}$ is affine.

By combining Proposition 3.1 and Proposition 2.6 we immediately get:

Corollary 3.2. Let $\left.v, w: \mathbb{C}^{N} \rightarrow\right] 0, \infty[$ be radial continuous weights, $1 \leq p, q<\infty$. Assume that $v$ is p-essential, $w$ is $q$-essential and that for some $\alpha>1$

$$
\lim _{r \rightarrow \infty} \frac{w(r)}{v(\alpha r)}=+\infty
$$

If $\psi \in \mathcal{H}\left(\mathbb{C}^{N} ; \mathbb{C}^{N}\right)$ satisfies that the composition operator $C_{\psi}: \mathcal{F}_{v}^{p} \rightarrow \mathcal{F}_{w}^{q}$ is continuous, then $\psi$ is affine. 
Example 3.3. The weights $v(z)=e^{-\gamma|z|^{2}-m \log |z|}$ and $w(z)=e^{-\beta|z|^{2}-k \log |z|}$ are $p$ essential for any $1 \leq p<\infty$ (see Example 1.3). Moreover condition (3.1) is satisfied, simply by choosing $\alpha>1$ such that $\alpha^{2} \gamma>\beta$. Hence we get that if a composition operator between Fock-Sobolev spaces is continuous, then $\psi$ is affine. Thus Corollary 3.2 covers some of the results in [13].

Corollary 3.4. Let $v, w: \mathbb{C} \rightarrow] 0, \infty[$ be radial continuous decreasing p-essential weights and assume that for some $\alpha>1$

$$
\lim _{r \rightarrow \infty} \frac{w(r)}{v(\alpha r)}=\infty
$$

Let $\psi \in \mathcal{H}(\mathbb{C})$ non-constant. The composition operator $C_{\psi}: \mathcal{F}_{v}^{p} \rightarrow \mathcal{F}_{w}^{p}$ is continuous if and only if

$$
\sup _{z \in \mathbb{C}} \frac{w(z)}{v(\psi(z))}<\infty
$$

In this case, $\psi$ is affine.

Proof. The necessity part follows from Proposition 2.6. Conversely, if $\sup _{z \in \mathbb{C}} \frac{w(z)}{v(\psi(z))}<$ $\infty$, then by Proposition 3.1, we get that $\psi(z)=a z+b$, with $a \neq 0$. Therefore, for every $f \in \mathcal{F}_{v}^{p}$ :

$$
\int_{\mathbb{C}}|f(\psi(z))|^{p} w^{p}(z) d z=\frac{1}{a} \int_{\mathbb{C}}|f(z)|^{p} w^{p}\left(\frac{z-b}{a}\right) d z \leq C^{p} \int_{\mathbb{C}}|f(z)|^{p} v^{p}(z) d z .
$$

Proposition 3.5. Let $\psi(z)=A z+b$, with $A$ a $N \times N$ complex matrix and $b \in \mathbb{C}^{N}$ and let $\left.v: \mathbb{C}^{N} \rightarrow\right] 0, \infty[$ be a radial continuous strictly decreasing weight.

(1) If $\| A||=\sup \{|A z|:|z|=1\}<1$, then $\sup _{z \in \mathbb{C}^{N}} \frac{v(z)}{v(A z+b)}<+\infty$.

(2) If $\|A\|>1$ and

$$
\forall \beta>1 \quad \lim _{r \rightarrow \infty} \frac{v(r)}{v(\beta r)}=+\infty
$$

then

$$
\sup _{z \in \mathbb{C}^{N}} \frac{v(z)}{v(A z+b)}=+\infty
$$

Proof Assume $v(z)=\exp (-\varphi(|z|))$, with $\varphi:[0, \infty[\rightarrow[0, \infty[$ continuous and strictly increasing.

(1) Set $\varepsilon=1-\|A\|$. For $|z|>\frac{|b|}{\varepsilon}$ we get

$$
\varphi(|A z+b|) \leq \varphi(|| A|| \cdot|z|+|b|) \leq \varphi(|| A|| \cdot|z|+\varepsilon|z|)=\varphi(|z|) .
$$

It is enough to take $C:=\max \left\{\varphi(|A z+b|):|z| \leq \frac{|b|}{\varepsilon}\right\}$ to get

$$
\varphi\left(|A z+b| \leq \varphi(|z|)+C, \quad z \in \mathbb{C}^{N} .\right.
$$

(2) Take $\varepsilon>0$ such that $1+2 \varepsilon<\|A\|$. There exists $\xi \in \mathbb{C}^{N}$ such that $|\xi|=1$ and $|A \xi| \geq 1+2 \varepsilon$. For every $n \in \mathbb{N}$ such that $n>\frac{|b|}{\varepsilon}$

$$
|A(n \xi)+b| \geq|n A \xi|-|b| \geq n(1+2 \varepsilon)-|b| \geq n(1+\varepsilon)+(n \varepsilon-|b|) \geq n(1+\varepsilon) .
$$

Assume that there exists $C>0$ such that

$$
\varphi(|A z+b|) \leq C+\varphi(|z|) \quad \forall z \in \mathbb{C}^{N} .
$$


Then, for every $n>\frac{|b|}{\varepsilon}$ :

$$
\varphi(n(1+\varepsilon)) \leq \varphi(|A(n \xi)+b|) \leq C+\varphi(n),
$$

hence

$$
\varphi(n(1+\varepsilon))-\varphi(n) \leq C
$$

This contradicts

$$
\lim _{r \rightarrow \infty} \varphi((1+\varepsilon) r)-\varphi(r)=+\infty
$$

We discuss now the case $N=1$ and $\psi(z)=a z+b$ with $|a|=1$. If $b=0$, then clearly $\frac{v(a z)}{v(z)}=1$ for every $z \in \mathbb{C}$. If $b \neq 0$, we consider some cases of particular interest.

Proposition 3.6. Let $\psi(z)=a z+b$ with $a \in \mathbb{C},|a|=1$ and $b \in \mathbb{C}, b \neq 0$. Let $v(z)=\exp (-\varphi(|z|))$, with $\varphi:[0, \infty[\rightarrow[0, \infty[$ continuous and strictly increasing. If

$$
\lim _{r \rightarrow \infty} \varphi(r+|b|)-\varphi(r)=+\infty,
$$

then

$$
\sup _{z \in \mathbb{C}} \frac{v(z)}{v(a z+b)}=+\infty
$$

In particular $\sup _{z \in \mathbb{C}} \frac{v(z)}{v(a z+b)}=+\infty$ if $v(z)=e^{-\alpha|z|^{p}}, p>1$, if $v(z)=e^{-e^{|z|}}$ or if $v(z)=e^{-|z|^{2}-m \log |z|}, m \in \mathbb{N}$.

Proof. Assume $a=e^{i t}$ and $b=|b| e^{i \theta}$. If $z=r e^{i(\theta-t)}$ with $r>0$, then

$$
|a z+b|=\left|(r+|b|) e^{i \theta}\right|=r+|b|,
$$

hence

$$
\lim _{|z| \rightarrow \infty} \varphi(|a z+b|)-\varphi(|z|)=+\infty
$$

and therefore $\sup _{z \in \mathbb{C}} \frac{v(z)}{v(a z+b)}=+\infty$.

If $\varphi(r)=\alpha r^{p}, p>1$, we have

$\alpha(r+|b|)^{p}=\alpha r^{p}\left(1+\frac{|b|}{r}\right)^{p} \geq \alpha r^{p}+\alpha p|b| r^{p-1} \geq \alpha r^{p}+\alpha p|b| r^{p-1}=\varphi(r)+\alpha p|b| r^{p-1}$.

Therefore

$$
\lim _{r \rightarrow \infty} \varphi(r+|b|)-\varphi(r)=+\infty .
$$

Consider $\varphi(r)=e^{r}$. Then $e^{r+|b|}=e^{|b|} e^{r}=e^{|b|} e^{r}$ and therefore $\lim _{r \rightarrow \infty} e^{r+|b|}-e^{r}=$ $\lim _{r \rightarrow \infty} e^{r}\left(e^{|b|}-1\right)=+\infty$.

Finally, if $\varphi(r)=r^{2}+m \log r$,

$$
\begin{aligned}
& \lim _{r \rightarrow \infty}\left((r+|b|)^{2}+m \log (r+|b|)-r^{2}-m \log r\right)= \\
= & \lim _{r \rightarrow \infty} 2|b| r+|b|^{2}+m \log \left(1+\frac{|b|}{r}\right)=+\infty .
\end{aligned}
$$


Example 3.7. Let $\psi(z)=a z+b$ with $a \in \mathbb{C},|a|=1$ and $b \in \mathbb{C}, b \neq 0$. Let $v(z)=\exp (-\varphi(|z|))$, with $\varphi(r)=r^{p}, 0<p \leq 1$. If $|z| \geq 1$ :

$$
\begin{aligned}
\varphi(|a z+b|) & =\alpha|a z+b|^{p} \leq \alpha(|z|+|b|)^{p}=\alpha|z|^{p}\left(1+\frac{|b|}{|z|}\right)^{p} \leq \\
& \leq \alpha|z|^{p}\left(1+p \frac{|b|}{|z|}\right)=\alpha|z|^{p}+\alpha p|b||z|^{p-1} \leq \varphi(|z|)+\alpha p|b| .
\end{aligned}
$$

Then

$$
\varphi(|a z+b|) \leq \varphi(|z|)+\alpha p|b|+\max _{|z| \leq 1} \varphi(|a z+b|) .
$$

Therefore $\sup _{z \in \mathbb{C}} \frac{v(z)}{v(a z+b)}<+\infty$. This should be compared with the behaviour of the weight when $p>1$.

Proposition 3.8. Let $\psi(z)=A z+b$ where $A$ is a $N \times N$ complex matrix, $b \in \mathbb{C}^{N}$, $1 \leq p \leq \infty, v(z)=\exp (-\varphi(|z|))$, with $\varphi:[0, \infty[\rightarrow[0, \infty[$ continuous and strictly increasing, such that $v$ is p-essential.

(1) If $\|A\|<1$, then $C_{\psi}: \mathcal{F}_{v}^{\infty} \rightarrow \mathcal{F}_{v}^{\infty}$ is continuous.

(2) If $\|A\|<1$ and for some $\alpha>1$

$$
\lim _{r \rightarrow \infty} \frac{v(r)}{v(\alpha r)}=\infty
$$

then $C_{\psi}: \mathcal{F}_{v}^{p} \rightarrow \mathcal{F}_{v}^{p}$ is continuous for any $1 \leq p<\infty$.

(3) If $\|A\|>1$ and

$$
\forall \beta>1 \quad \lim _{r \rightarrow \infty} \frac{v(r)}{v(\beta r)}=+\infty,
$$

then $C_{\psi}: \mathcal{F}_{v}^{p} \rightarrow \mathcal{F}_{v}^{p}$ is not continuous for any $1 \leq p \leq \infty$.

(4) If $N=1, \psi(z)=a z+b,|a|=1$, and

$$
\lim _{r \rightarrow \infty} \varphi(r+|b|)-\varphi(r)=+\infty,
$$

then $C_{\psi}: \mathcal{F}_{v}^{\infty} \rightarrow \mathcal{F}_{v}^{\infty}$ is continuous if and only if $b=0$.

(5) If $N=1, \psi(z)=a z+b,|a|=1$, and (3.7) holds, then $C_{\psi}: \mathcal{F}_{v}^{p} \rightarrow \mathcal{F}_{v}^{p}$ is continuous if and only if $b=0$, for any $1 \leq p<\infty$.

Proof. It is enough to apply Proposition 3.5, recalling that, for $p=\infty C_{\psi}$ is continuous if and only if $\sup _{z \in \mathbb{C}^{N}} \frac{v(z)}{v(A z+b)}<\infty$ (see [7]) and for $1 \leq p<\infty$, if $C_{\psi}$ is continuous then $\sup _{z \in \mathbb{C}^{N}} \frac{v(z)}{v(A z+b)}<\infty$ and the converse holds if for some $\alpha>1$ $\lim _{r \rightarrow \infty} \frac{v(r)}{v(\alpha r)}=+\infty$. If (3.7) holds, this condition is satisfied for any $\alpha>1$, since $\varphi(\alpha r)-\varphi(r) \geq \varphi(r+|b|)-\varphi(r)$ for $r>\frac{|b|}{\alpha-1}$.

Example 3.9. If $p=\infty$, Proposition 3.8 can be applied when $\varphi(r)=r^{q}, q>0$, $\varphi(r)=r^{2}+m \log r, m \in \mathbb{N}$ or $\varphi(r)=e^{r}$, since $e^{-\varphi}$ is essential by [8, Remark 3.11] and by [4, Corollary 16].

If $1 \leq p<\infty$, Proposition 3.8 can be applied when $\varphi(r)=\alpha r^{2}$ or $\varphi(r)=r^{2}+m \log r$ by example 2.3 .

We conclude with a result about composition operators acting between $\mathcal{F}_{v}^{2}$ spaces with different radial weights. The proof is based on the techniques of [25, Lemma 6.1]. 
Assume that $v$ is a continuous weight on $\mathbb{C}$ such that $\int_{0}^{\infty} r^{n} v(r) d r<\infty$ for every $n \in \mathbb{N}$. If we set $a_{n}=\int_{0}^{\infty} r^{2 n+1} v^{2}(r) d r$, then the reproducing kernel in $\mathcal{F}_{v}^{2}(\mathbb{C})$ is given by

$$
K_{z}(w)=\sum_{n=0}^{\infty} a_{n} \bar{z}^{n} w^{n}, \quad w \in \mathbb{C}
$$

and

$$
\left\|K_{z}\right\|_{2, v}=\left(\sum_{n=0}^{\infty} a_{n}|z|^{2 n}\right)^{\frac{1}{2}}
$$

(see e.g. $[5,6,25]$ ).

Theorem 3.10. Let $v$ and $w$ be decreasing continuous radial weights on $\mathbb{C}$ such that $a_{n}=\int_{0}^{\infty} r^{n} v(r) d r<+\infty, b_{n}=\int_{0}^{\infty} r^{n} w(r) d r<+\infty$ for every $n \in \mathbb{N}$. Assume that $M:=\sup _{n} \sqrt[2 n]{b_{n} / a_{n}}<+\infty$. Let $\psi \in \mathcal{H}(\mathbb{C})$. If the composition operator $C_{\psi}$ : $\mathcal{F}_{v}^{2}(\mathbb{C}) \rightarrow \mathcal{F}_{w}^{2}(\mathbb{C})$ is continuous, then $\psi$ is affine.

Proof. As in the proof of Theorem 2.6, we get that, if $C_{\psi}$ is continuous, then there exists $C \geq 1$ such that

$$
\forall z \in \mathbb{C}^{N} \quad\left\|\delta_{\psi(z)}\right\|_{\left(\mathcal{F}_{v}^{2}\right)^{\prime}} \leq C\left\|\delta_{z}\right\|_{\left(\mathcal{F}_{w}^{2}\right)^{\prime}},
$$

or equivalently

$$
\forall z \in \mathbb{C}^{N} \quad\left\|K_{\psi(z)}\right\|_{2, v} \leq C\left\|K_{z}\right\|_{2, w} .
$$

Therefore we can apply (3.9) to get, for each $z \in \mathbb{C}$,

$$
\left(\sum_{n=0}^{\infty} a_{n}|z|^{2 n}\left(\frac{|\psi(z)|^{2 n}}{|z|^{2 n}}-C^{2} \frac{b_{n}}{a_{n}}\right)\right)=\left(\sum_{n=0}^{\infty} a_{n}|\psi(z)|^{2 n}-C^{2} \sum_{n=0}^{\infty} b_{n}|z|^{2 n}\right) \leq 0 .
$$

It is enough to prove that $\sup _{|z| \geq 1} \frac{|\psi(z)|}{|z|}<\infty$. Otherwise, there would exist a sequence $\left(z_{k}\right)_{k}$ of complex numbers such that $\lim _{k \rightarrow \infty}\left|z_{k}\right|=\infty$ and $\lim _{k \rightarrow \infty} \frac{\left|\psi\left(z_{k}\right)\right|}{\left|z_{k}\right|}=$ $\infty$. Then there would exist $\nu \in \mathbb{N}$ such that for every $k \geq \nu$ :

$$
\frac{\left|\psi\left(z_{k}\right)\right|}{\left|z_{k}\right|} \geq C^{2} \cdot(M+1),
$$

with $M>0$ as in the statement. Now, for every $k \in \mathbb{N}$ and $k \geq \nu$ we have

$$
\frac{\left|\psi\left(z_{k}\right)\right|^{2 n}}{\left|z_{k}\right|^{2 n}} \geq C^{2 n} \cdot(M+1)^{2 n} \geq C^{2}\left(M^{2 n}+1\right) \geq C^{2}\left(\frac{b_{n}}{a_{n}}+1\right),
$$

hence

$$
\begin{aligned}
0<C^{2} a_{1}\left|z_{k}\right| & \leq \sum_{n=0}^{\infty} a_{n}\left|z_{k}\right|^{n}\left(C^{2}\left(\frac{b_{n}}{a_{n}}+1\right)-C^{2} \frac{b_{n}}{a_{n}}\right) \\
& \leq \sum_{n=0}^{\infty} a_{n}\left|z_{k}\right|^{n}\left(\frac{\left|\psi\left(z_{k}\right)\right|^{2 n}}{\left|z_{k}\right|^{2 n}}-C^{2} \frac{b_{n}}{a_{n}}\right) \leq 0 .
\end{aligned}
$$

So we reach a contradiction.

Corollary 3.11. Let $v$ be a decreasing continuous weight on $\mathbb{C}$ such that $\int_{0}^{\infty} r^{n} v(r) d r<$ $\infty$ and let $\psi \in \mathcal{H}(\mathbb{C})$. If the composition operator $C_{\psi}: \mathcal{F}_{v}^{2}(\mathbb{C}) \rightarrow \mathcal{F}_{v}^{2}(\mathbb{C})$ is continuous, then $\psi$ is affine. 
Remark 3.12. Observe that Corollary 3.11 applies also to the weights considered in Example 2.4 and, more in general, those in [14], although they are not 2-essential.

Acknowledgements. The research of Bonet was partially supported by the projects MTM2016-76647-P and GV Prometeo/2017/102 (Spain).

\section{REFERENCES}

[1] W. Al-Rawashden: Compact Weighted Composition Operators Between Generalized Fock Spaces, Missouri J. Math. Sci. 28(1), 62-75 (2016)

[2] S. Bargmann: On a Hilbert space of analytic functions and an associated integral transform, Commun. Pure Appl. Math. 24, 187-214 (1961)

[3] K.D. Bierstedt, J. Bonet, And A. Galbis: Weighted spaces of Holomorphic functions on balanced domains, Michigan Math. J., 40(2), 271-297 (1993)

[4] K.D. Bierstedt, J. Bonet, And J. Taskinen: Associated weights and spaces of holomorphic functions, Studia Math. 127, 137-168 (1998)

[5] O. Blasco: Boundedness of Volterra operators in spaces of entire functions, Ann. Acad. Sci. Fenn. Math. 43 (1) , 89-107 (2018)

[6] O. Blasco: Operators on Fock-type and weighted spaces of entire functions, Preprint (2018)

[7] J. Bonet, M. Fritz, AND E. JoRdA: Composition operators between weighted inductive limits of spaces of holomorphic functions, Publ. Math. 67 (3), 333-348 (2005)

[8] J.Bonet And J. Taskinen: A note about Volterra operators on weighted Banach spaces of entire functions, Math. Nachr. 288, 1216-1225 (2015)

[9] A. Borichev: The polynomial approximation property in Fock-type spaces, Math. Scand. 82, 256-264 (1998).

[10] B. Carswell, B. MacCluer, and A. Schuster: Composition operators on the Fock space, Acta Sci. Math. (Szeged), 69, 871-887 (2003)

[11] C. Cascante, J. Fábrega, and J. Peláez, Littlewood-Paley formulas and Carleson measures for weighted Fock spaces induced by $A^{\infty}$-type weights, Potential Anal. https://doi.org/10.1007/s11118-018-9680-z (2018)

[12] H. R. Сно And K. Zhu: Fock-Sobolev spaces and their Carleson measures, J. Funct. Anal. 263, 2483-2506 (2012)

[13] H. R. Cho, B.R. Choe, And H. Koo: Linear combination of composition operators on the Fock-Sobolev spaces, Potential Anal. 4 (4), 1223-1246 (2014)

[14] O. Constantin and J. A.PelÁEz: Integral operators, Embedding theorems and a LittlewoodPaley formula on Fock spaces, J. Geom. Anal. 26 (2), 1109-1154 (2016)

[15] G. B. Folland: Harmonic Analyis in Phase spaces, Princeton University Press (1989)

[16] A. Galbis: Weighted Banach spaces of entire functions, Archiv Math. 62, 58-64 (1994)

[17] T. LE: Normal and isometric weighted composition operators on the Fock space, Bull. London Math. Soc. 46 (4), 847-856 (2014)

[18] W.Lusky, On the isomorphism classes of weighted spaces of harmonic and holomorphic functions, Studia Math. 175, 19-45, (2006)

[19] N. Marco, M. Massaneda, And J. Ortega-Cerdì: Interpolating and sampling sequences for entire functions, Geom. Funct. Anal. 13 , 862-914 (2003)

[20] J. Ortega-Cerdì: Sampling measures Publ. Mat. 42 (2), 559-566 (1998)

[21] I. Segal: Mathematical problems of relativistic physics, in Kac, M., Proceedings of the Summer Seminar, Boulder, Colorado, 1960, Vol. II, Lectures in Applied Mathematics, American Mathematical Society (1963)

[22] K. Seip And E. H. Youssfi: Hankel Operators on Fock Spaces and related Bergman kernel estimates, J. Geom. Anal. 23, 170-201 (2013)

[23] A. P. Schuster And D. Varolin: Toeplitz Operators and Carleson Measures on Generalized Bargmann-Fock Spaces, Integr. Equ. Oper. Theory 72, 363-392 (2012)

[24] S. Stevic: Weighted composition operators between Fock-type spaces in $C^{N}$, Appl Math. Comput. 215, 2750-2760 (2009)

[25] J. Stochel and J. B. Stochel: Composition operators on Hilbert spaces of entire functions with analytic symbols, J. Math. Anal. Appl. 454, 1019-1066 (2017) 
[26] P.T.Tien and L.H. Khoi: Weighted composition operators between different Fock spaces, Potential Anal. https://doi.org/10.1007/s11118-017-9678-y (2018)

[27] S. UeKI: Weighted composition operator on the Fock space, Proc. Amer. Math. Soc. 35, 1405$1410(2007)$

[28] S. UEKI: Weighted composition operators on the Bargmann-Fock spaces, International Journal of Modern Mathematics 3, 231-243 (2008).

[29] S. UeKI: Weighted composition operators on some function spaces of entire functions, Bull. Belg. Math. Soc. Simon Stevin 17 , 343-353 (2010)

[30] K. ZHu: Analysis on Fock Spaces, Springer-Verlag, New York, 2012.

José Bonet, Instituto Universitario de Matemática Pura y Aplicada, Universitat Politècnica de València, E-46071 Valencia, Spain

E-mail address: jbonet@mat.upv.es

Elisabetta M. Mangino, Dipartimento di Matematica e Fisica "E.De Giorgi", UniverSità del Salento, I-73100 Lecce, Italy

E-mail address: elisabetta.mangino@unisalento.it 